\title{
Focos de investigación sobre aprendizaje universitario en el área de dirección de operaciones
}

\author{
Juan A. Marin-Garcia ${ }^{a}$ \\ ${ }^{a}$ ROGLE Research Group (DOE Universitat Politècnica de València, jamarin@omp.upv.es)
}

\begin{abstract}
What are the topics that higher education professors of the area of operations management in Spain are researching about learning of their students in recent years? Are the approaches that support these investigations adequate?

We aim to collect and summarize the main works on learning research, carried out by members of ACEDEDOT and published in the last year (2017), identifying themes and focus on the type of student learning. With this we intend to create a map of the current situation and propose possible suggestions to build an evidence-based operations management teaching.
\end{abstract}

Keywords: scholarship; student learning; student engagement

\begin{abstract}
Resumen
En esa investigación analizamos cuales son los focos de investigación sobre aprendizaje de los profesores universitarios de dirección de operaciones españoles y reflexionamos sobre si las preguntas de investigación abordadas son las más adecuadas.

Nos centraremos en las investigaciones llevadas a cabo por las personas vinculadas a la asociación científica ACEDEDOT y publicadas en el año 2017. Identificaremos los temas y enfoques de aprendizaje abordados. Con ello pretendemos dibujar un mapa del estado actual de la investigación en el área y plantear sugerencias para hacer avanzar hacia una docencia universitaria de dirección de operaciones basada en evidencias.
\end{abstract}

Palabras clave: docencia basada en evidencias; aprendizaje de los estudiantes; implicación de los estudiantes en el aprendizaje.

\section{Introducción}

La docencia basada en la evidencia de qué es lo que mejor favorece el aprendizaje de nuestros alumnos (Burke-Smalley, 2014; Cascio, 2007; Morrell \& Learmonth, 2015) es una corriente de trabajo e investigación que poco a poco va ganando terreno y se relaciona con la base del movimiento creado bajo la etiqueta de scholarship (Boyer, 1990; Delbecq, 2007; Fernandez March, 2008; Pearce, 2007).

Todo esto se ha visto condicionado por la evolución del significado e implicaciones de los niveles de concepción docente (Biggs \& Tang, 2011 (1st edition 1999); Ramsden, 1992) y los enfoques de aprendizaje de los estudiantes (superficial, estratégico y profundo) (Marton et al., 2005; Paricio Royo, 2013; Paricio Royo \& Allueva Pinilla, 2011). Todos estos 
desarrollos clásicos en la pedagogía han estado marcando la metodología y enfoque de la investigación sobre aprendizaje de finales del siglo pasado y principio de este.

Sin embargo, ¿estamos aprovechando los docentes de Dirección de Operaciones estos desarrollos? ¿Qué estamos investigando en el último año los profesores universitarios del área en España? ¿̇Son adecuados los planteamientos que sustentan nuestras investigaciones?

Nuestra investigación pretende recoger y resumir los principales trabajos sobre investigación de aprendizaje, realizados por miembros de ACEDEDOT en el año 2017, identificando temáticas, métodos, niveles de concepción docente y enfoque sobre tipo de aprendizaje de los alumnos. Con ellos pretendemos crear un mapa de la situación actual y plantear posibles sugerencias para ir construyendo una docencia de dirección de operaciones basada en evidencias.

\section{Objetivos}

Identificar los focos de investigación sobre aprendizaje de los trabajos publicados, en revistas científicas revisadas por pares, por profesores de Dirección de Operaciones que imparten asignaturas universitarias en España (en grado o máster).

\section{Desarrollo de la innovación}

En este trabajo vamos a trabajar con dos dimensiones que nos permitirán identificar los focos de investigación (Figura 1). Por un lado, nos centraremos en el punto de vista del investigador/profesor y analizaremos el nivel de conceptualización docente que sustenta sus preguntas de investigación (Biggs \& Tang, 2011 (1st edition 1999); Ramsden, 1992). Como nivel 1 caracterizaremos las preguntas de investigación que intentan identificar el mejor método/recurso para lograr el aprendizaje de los alumnos. El nivel 2 agrupa las preguntas relacionadas con cómo enfocan el aprendizaje los alumnos. El nivel 3 centra sus preguntas en identificar, en contextos específicos, en qué consiste un buen aprendizaje y qué están aprendiendo los alumnos.

Por otro lado, una de las variables que mejor explica la variabilidad de los resultados o las conductas de los estudiantes son los enfoques de aprendizaje que presenta el alumno (Marton et al., 2005). Por ello, analizaremos cuales son los que están presentes, explícita o implícitamente en las investigaciones publicadas. Los enfoques de aprendizaje que eligen los estudiantes pueden clasificarse en tres (Coffey \& Gibbs, 2002; Gibbs \& Coffey, 2000; Marton et al., 2005; Paricio Royo, 2013; Paricio Royo \& Allueva Pinilla, 2011; Ramsden, 1992): superficial (almacenar información inconexa de manera rutinaria), estratégico (hacer las tareas o mostrar los comportamientos que son calificados favorablemente por el profesor, para obtener la máxima nota posible, con el mínimo esfuerzo necesario para ello) y profundo (implicarse apasionadamente en el aprendizaje dando sentido a los conceptos de la materia y vinculándolos al desarrollo personal). Las categorías de los enfoques de aprendizaje tienen una relación directa con las categorías en las que se pueden agrupar las concepciones del aprendizaje, las concepciones de la enseñanza o los resultados de aprendizaje. Por ejemplo, el enfoque estratégico suele exigir al alumno, en casi todas las

(cc) EY-NC-ND 2018, Universitat Politècnica de València

Congreso IN-RED (2018) 
asignaturas universitarias, que demuestre unas capacidades o que pueda aplicar un conocimiento en un contexto conocido (preguntas tipo de examen o ejercicios parecidos a los realizados en clase o ajustarse a lo que se muestra en las rúbricas de corrección), pero normalmente no van más allá de presentar una respuestas coherentes con escasas argumentaciones pues no han invertido tiempo en intentar comprender profundamente.

En principio, las investigaciones bajo cualquiera de los niveles de conceptualización pueden estar relacionadas con cualquiera de los enfoques de aprendizaje. Así, por ejemplo, una investigación de nivel 3 puede plantearse identificar cuáles son los resultados de aprendizaje de los estudiantes y si estos reproducen información (superficial) o son capaces de comprender (profundo). Del mismo modo una investigación de nivel 1 puede plantearse cuál es el mejor método para logra aprendizaje profundo en los alumnos.

En la investigación educativa se ha avanzado mucho en lo general y poco en lo específico. Es decir, hay mucho camino andado en los niveles 1 y 2 de conceptualización docente, pero es preciso seguir investigando en preguntas relacionadas con el nivel 3. Debemos intentar conocer los factores externos e internos que condicionan el aprendizaje de una materia en un contexto determinado. Para ello, es imprescindible el trabajo de los profesores investigando desde sus didácticas específicas (Fernandez March, 2008; Fernández March, 2010).

\begin{tabular}{|c|c|c|c|c|}
\hline $\begin{array}{l}\text { Nivel de } \\
\text { conceptualización } \\
\text { docente }\end{array}$ & $\begin{array}{l}\text { Enfoques de } \\
\text { aprendizaje }\end{array}$ & $\begin{array}{l}\text { Concepción } \\
\text { del } \\
\text { aprendizaje }\end{array}$ & $\begin{array}{l}\text { Concepción } \\
\text { de la } \\
\text { enseñanza }\end{array}$ & $\begin{array}{ll}\text { Resultados de } & \text { de } \\
\text { aprendizaje } & \end{array}$ \\
\hline $\begin{array}{l}\text { Nivel } 1 \\
\text { ¿Cómo enseño? } \\
\text { Centrado en la } \\
\text { docencia. ¿cuál es } \\
\text { el mejor método } \\
\text { para...? }\end{array}$ & Superficial & $\begin{array}{l}\text { Almacenar } \\
\text { información o } \\
\text { aumentar el } \\
\text { conocimiento } \\
\text { de forma } \\
\text { rutinaria }\end{array}$ & $\begin{array}{l}\text { Impartir } \\
\text { información / } \\
\text { Transmitir } \\
\text { conocimiento }\end{array}$ & $\begin{array}{l}\text { Listas } \\
\text { incoherentes de } \\
\text { información. } \\
\text { Descripciones } \\
\text { breves } \\
\text { reproducidas }\end{array}$ \\
\hline $\begin{array}{l}\text { Nivel } 2 \\
\text { ¿Cómo aprenden? } \\
\text { Centrado en el } \\
\text { estudiante ¿qué } \\
\text { hacen los } \\
\text { estudiantes o de } \\
\text { qué depende que } \\
\text { elijan un enfoque } \\
\text { de aprendizaje u } \\
\text { otro? }\end{array}$ & Estratégico & $\begin{array}{l}\text { Aplicar } \\
\text { conocimiento } \\
\text { y capacidades }\end{array}$ & $\begin{array}{l}\text { Dirigir el } \\
\text { aprendizaje } \\
\text { activo }\end{array}$ & $\begin{array}{ll}\text { Esbozo } & \text { de } \\
\text { respuestas } & \\
\text { coherentes } & \text { sin } \\
\text { argumentar } & \end{array}$ \\
\hline $\begin{array}{l}\text { Nivel } 3 \\
\text { ¿Qué aprenden? } \\
\text { Centrado en el } \\
\text { estudiante. ¿qué } \\
\text { significa } \\
\text { aprender/compren } \\
\text { der en el contexto } \\
\text { de mi asignatura y } \\
\text { cómo puedo } \\
\text { medirlo? }\end{array}$ & Profundo & $\begin{array}{l}\text { Dar sentido a } \\
\text { las ideas y } \\
\text { desarrollarse } \\
\text { personalment } \\
\text { e }\end{array}$ & $\begin{array}{lr}\text { Facilitar la } \\
\text { comprensión } \\
\text { profunda y } \\
\text { potenciar el } \\
\text { cambio } \\
\text { conceptual }\end{array}$ & $\begin{array}{l}\text { Explicaciones } \\
\text { completas } \\
\text { argumentadas. } \\
\text { Concepciones } \\
\text { individuales } \\
\text { sobre el tema. } \\
\text { Pensar como } \\
\text { piensan los } \\
\text { profesionales }\end{array}$ \\
\hline
\end{tabular}


Hemos seleccionado para el análisis sólo las publicaciones en revistas científicas arbitradas por pares. Los trabajos en congresos suelen adolecer de diseños metodológicos incompletos y mostrar planteamientos preliminares que, en caso de resultar interesantes, pueden ser enriquecidos con las opiniones recibidas en el congreso para convertirlos en envíos a revistas. Consideramos que los trabajos que han finalizado su recorrido académico en la fase de congreso y no se han llevado a revistas, son trabajos incompletos o intranscendentes o con escaso potencial y, por lo tanto, sus autores deciden no dedicar el esfuerzo necesario para elevarlos a una conversación académica de alto nivel (como la que puede propiciarse en el proceso de revisión y posterior divulgación en revistas científicas).

La disciplina académica de dirección de operaciones se enmarca (en España) en el campo científico de las Ciencias Empresariales. Las asignaturas de esta disciplina suelen estar presentes sólo en los grados o máster de Administración de empresas y en alguna de las ingenierías de la rama industrial (especialmente en el Grado en Ingeniero de Organización o el master ingeniero industrial). Quizás por ello, los profesores universitarios de una asignatura de dirección de operaciones suelen tener una formación básica de Administración de Empresas o de Ingeniería. En España hay dos asociaciones científicas en las que se suelen dar cita los profesores universitarios de Dirección de Operaciones. Por un lado, podemos señalar a ACEDEDOT, que es la sección de operaciones y tecnología de la asociación científica de economía y dirección de empresas (ACEDE). Por otro, la asociación para el desarrollo de la ingeniería de organización (ADINGOR). Aunque algunos profesores están presentes en ambas asociaciones, en general podemos considerar que, en ACEDEDOT, predominan los profesores con perfil más de administración de empresas y más vinculados a ciencias sociales (aunque también haya ingenieros o personas del área de investigación operativa); mientras que en ADINGOR hay más presencia de ingenieros.

Para este trabajo, seleccionaremos los artículos, relacionados con aprendizaje universitario, publicados en 2017 por profesores adscritos a ACEDEDOT (pudiendo extender en investigación futura a otros años recientes y a profesores adscritos a ADINGOR).

Hemos contactado por mail o personalmente con los profesores de la sección solicitándoles que nos envíen los trabajos publicados sobre investigación en aprendizaje. Los trabajos recibidos se han revisado para comprobar que cumplían los criterios para ser seleccionados en esta revisión (ver Tabla 1)

Tabla 1.- Criterios de inclusión y exclusión

\begin{tabular}{|l|l|}
\hline $\begin{array}{l}\text { Criterios } \\
\text { de } \\
\text { inclusión }\end{array}$ & $\begin{array}{l}\text { - Publicados en revistas científicas } \\
\text { - Durante el año } 2017 \\
\text { Investigación cuya contribución principal es sobre aprendizaje de estudiantes } \\
\text { universitarios }\end{array}$ \\
$\begin{array}{l}\text { Al menos uno de los autores pertenece a ACEDEDOT y sus líneas de } \\
\text { investigación y/o docencia son de dirección de operaciones }\end{array}$ \\
\hline $\begin{array}{l}\text { Criterios } \\
\text { de }\end{array}$ & $\begin{array}{l}\text { Contribuciones a congresos, tesis, entradas de blog u otro material no } \\
\text { publicado }\end{array}$ \\
\hline
\end{tabular}

(cc) EY-NC-ND 2018, Universitat Politècnica de València

Congreso IN-RED (2018) 


\begin{tabular}{|l|l|}
\hline exclusión & $\begin{array}{l}\text { Investigación sobre aspectos de dirección de operaciones cuya contribución } \\
\text { no es aprendizaje }\end{array}$ \\
& $\begin{array}{l}\text { La contribución no es aplicable a estudiantes universitarios (solo se centran en } \\
\text { profesionales) }\end{array}$
\end{tabular}

Cada uno de los trabajos seleccionados se ha codificado extrayendo la siguiente información:

- Área académica de cada uno de los autores: marcando como “dirección de operaciones” u “otros” en función de las principales publicaciones del autor, indexadas en Scopus o Web Of Science o Google Scholar, en los últimos 3 años ( 2014-2017)

- Preguntas de investigación que abordan en el artículo: las preguntas o contribución establecida explícitamente en el trabajo

- Para cada contribución o pregunta de investigación

o Nivel de conceptualización docente: nivel1, nivel2, nivel3, no definido

o Enfoques de aprendizaje: superficial, estratégico, profundo, no definido

- Información de contexto:

o Nivel académico donde se enmarca la contribución: $1^{\circ} / 2^{\circ}$ de grado, $3^{\circ} / 4^{\circ}$ de grado, máster, no definido

o Asignatura objeto de estudio: en el caso de ser una investigación centrada en una asignatura concreta

o Tamaño medio de grupo (alumnos matriculados por grupo): pequeño (menos de 20 alumnos); mediano (mas de 20 y menos de 60); grande (más de 60 alumnos); no definido

o Nombre de la titulación en la que se enmarca la/las asignaturas

o Universidad

El proceso de codificación y análisis ha sido realizado con la ayuda del programa Atlas.ti (Friese, 2012; Marin-Garcia, 2007)

\section{Resultados}

En los 22 trabajos analizados nos encontramos abundante desinformación para contextualizar las experiencias. Algunos trabajos (entre 4 y 6 según la variable a considerar) tienen un enfoque genérico y no se circunscriben a un contexto concreto (universidad, grado, asignatura, año de estudios o tamaño del grupo), pues su contribución, en principio, se plantea como generalizable a cualquiera de esos contextos. Sin embargo, el resto de los trabajos hacen referencia a una experiencia concreta. Siete de ellos hacen explícito que se contextualiza en el grado de dirección de empresas, otros dos se refieren a ingeniería electrónica, uno a ingeniería aeroespacial y los otros tres con información sobre el grado son posgrados (un master en economía, otro en dirección de empresas y un especialista universitario en producción ajustada). Respecto a las asignaturas, 7 son de dirección de operaciones, 1 de cadena de suministro, otra de producción ajustada, otra de mejora de procesos, dos sobre trabajos final de grado o máster y las otras dos son de 
organización de empresas. Dada los criterios de selección no es de extrañar que abunden las titulaciones relacionadas con administración de empresas y que la mayoría, de las que aportan información, se centren en asignatura vinculadas a dirección de operaciones. No hay demasiada información sobre el tamaño de grupo, pero cuando la aportan, la investigación sobre aprendizaje se suele realizar en asignaturas con grupos pequeños.

Las preguntas de investigación abordadas por los trabajos analizados se centran, principalmente, en enfoques de conceptualización de nivel 1 (Figura 2). 26 de las 31 preguntas identificadas se centran en lo que hace el profesor, y buscan justificar la "mejor forma de lograr algo”, bien sea la mejor metodología para lograr un resultado de aprendizaje de los alumnos o el mejor método para evaluar lo que los alumnos han adquirido ( $\mathrm{N}$ es mayor que 22 pues algunos trabajos tienen varias preguntas/contribuciones, cada una de ellas en el mismo o diferente nivel de conceptualización o enfoque de aprendizaje). Sólo en 5 casos hemos detectado indicios de que la contribución se centra en el estudiante, bien en cómo aprenden o en qué sería adecuado que aprendieran en la asignatura.

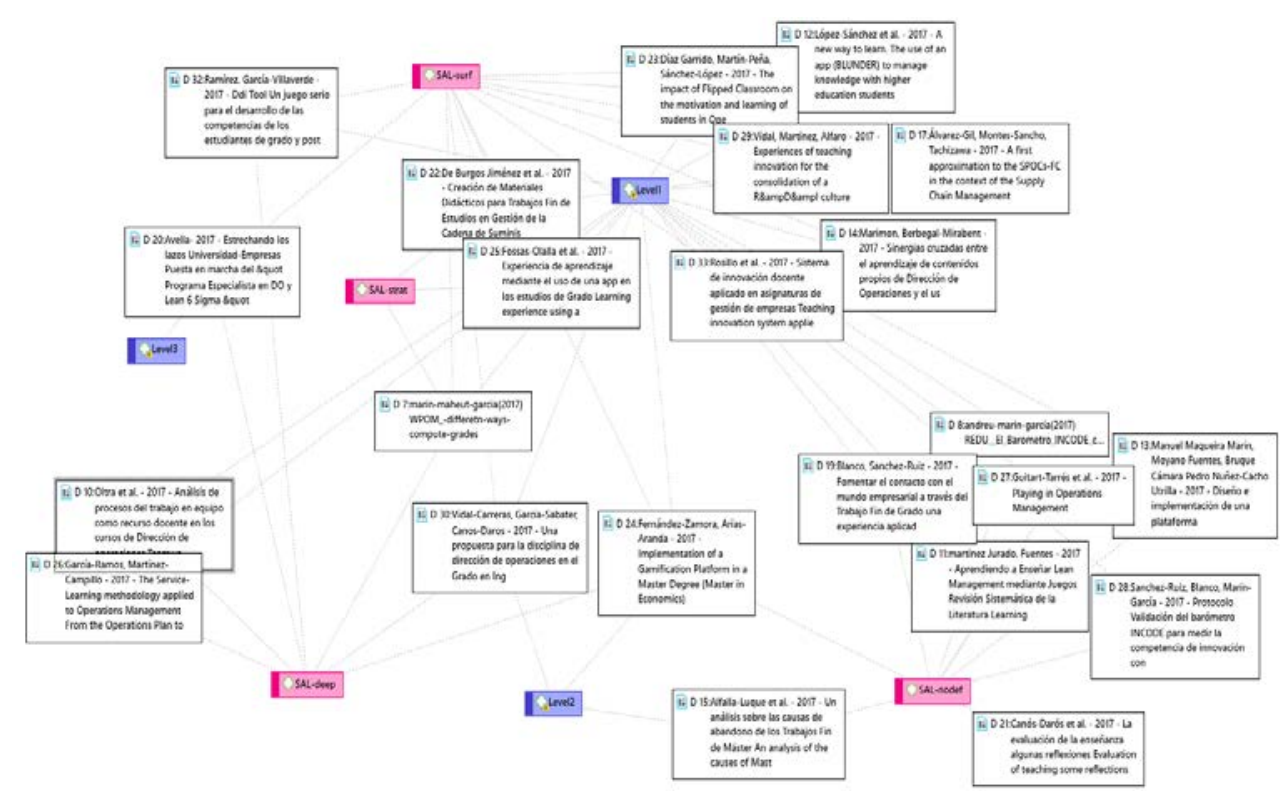

Figura 2.- Niveles de conceptualización y enfoques de aprendizaje

En la Tabla 2 podemos observar como la mayoría de los trabajos tienen un nivel 1 de conceptualización docente $\mathrm{y}$, al mismo tiempo analizan un enfoque de aprendizaje superficial. No obstante, 4 de los trabajos con nivel 1, buscan reflexionar sobre aprendizajes profundos de los alumnos y otros tres sobre aspectos que se podrían asociar a aprendizaje estratégico. Aparte de eso, 9 preguntas de investigación no hemos podido asociarlas a ningún enfoque de aprendizaje concreto, debido a falta de información.

(cc) EY-NC-ND 2018, Universitat Politècnica de València

Congreso IN-RED (2018) 
Tabla 2.-Trabajos y numero de preguntas/contribuciones por nivel de conceptualización y enfoques de aprendizaje

\begin{tabular}{|l|l|l|l|l|l|l|l|}
\hline ID & Level1 & Level2 & Level3 & $\begin{array}{l}\text { SAL- } \\
\text { surf }\end{array}$ & $\begin{array}{l}\text { SAL- } \\
\text { strat }\end{array}$ & SAL-deep & SAL-nodef \\
\hline (Alfalla-Luque et al., 2017) & & 1 & & & & & 1 \\
\hline (Alhely et al., 2017) & & & & & & & 1 \\
\hline (Álvarez-Gil et al., 2017) & 2 & & & 2 & & & \\
\hline (Andreu-Andrés et al., 2017) & 1 & & & & & & 1 \\
\hline (Avella Camarero, 2017) & & & 1 & 1 & & 2 & \\
\hline (Blanco \& Sanchez-Ruiz, 2017) & 1 & & & & & & 1 \\
\hline (Canós-Darós et al., 2017) & & & & & & & 1 \\
\hline (De Burgos Jiménez et al., 2017) & 1 & & & 1 & 1 & & \\
\hline (Díaz Garrido et al., 2017) & 1 & & & 1 & & & \\
\hline (Fernández-Zamora \& Arias-Aranda, 2017) & 3 & 2 & & 2 & & 3 & 1 \\
\hline (Fossas-Olalla et al., 2017) & 1 & & & 1 & 2 & & \\
\hline (García-Ramos \& Martínez-Campillo, 2017) & 1 & & & & & 1 & \\
\hline (Guitart-Tarrés et al., 2017) & 1 & & & & & & 1 \\
\hline (López-Sánchez et al., 2017) & 1 & & & 2 & & & \\
\hline (Maqueira Marín et al., 2017) & 1 & & & & & & 1 \\
\hline (Marimon \& Berbegal-Mirabent, 2017) & 1 & & & 1 & & & \\
\hline (Marin-Garcia et al., 2017) & 2 & & & 2 & 2 & 2 & \\
\hline (Martínez Jurado \& Moyano Fuentes, 2017) & 1 & & & & & & 1 \\
\hline (Oltra Mestre et al., 2017) & 1 & & & & & 1 & \\
\hline (Ramírez \& García-Villaverde, 2017) & 1 & & & 1 & & 1 & \\
\hline (Rosillo et al., 2017) & 2 & & & 1 & & & \\
\hline (Sanchez-Ruiz et al., 2017) & 1 & & & & & & 1 \\
\hline (Vidal et al., 2017) & 2 & & & 1 & & & \\
\hline (Vidal-Carreras et al., 2017) & 1 & 1 & & 2 & & 1 & \\
\hline Totales & 26 & 4 & 1 & 18 & 5 & 11 & 10 \\
\hline
\end{tabular}

A modo de ejemplo, presentamos en la Tabla 3 cómo se podría transformar una pregunta de investigación de nivel 1 en una batería de preguntas que situarían el foco de la investigación en una conceptualización docente de nivel 2 o nivel 3.

Tabla 3.- Ejemplo de propuesta de reformulación de preguntas de investigación para pasar de nivel 1 a nivel 2 y nivel 3 de conceptualización

En lugar de... (nivel 1) ¿la gamificación es una buena herramienta docente?, o ¿̇la gamificación sirve para que los alumnos aprendan más?

\section{... Formularlo de este modo (nivel 2 y nivel 3)}

¿Qué ocurre con los estudiantes de la asignatura xxxx del curso 2017/2018 cuando se enfrentan a la actividad YYYY (o conjunto de actividades) gamificadas? ¿Se estresan más o se motivan más? ¿sólo se genera motivación extrínseca o esa motivación les hace invertir más horas y, además, aumentar la motivación intrínseca? ¿Se orientan sólo a los resultados que resalta el panel de gamificación, o desarrollan un aprendizaje profundo y de alta intensidad respecto a los objetivos del curso y amplían, además, sus objetivos personales? ¿Qué tipo de objetivos/resultados de aprendizaje ayuda a construir esta experiencia de gamificación? 


\section{Conclusiones}

Tras los análisis realizados, parece que la mayoría de la investigación se queda en un nivel de conceptualización muy básico, que reproduce el tipo de investigaciones habituales antes de 1980. Este tipo de preguntas se consideran desfasadas pues aspiran a encontrar una "silver bullet" que funcione de manera exitosa en cualquier contexto y para cualquier estudiante. Es decir, que si los profesores elegimos el método o actividad correcta, nuestros estudiantes lograrán los resultados de aprendizaje esperados. Como si el problema consistiera en identificar el botón o palanca que activa en todos nuestros estudiantes el proceso de aprendizaje que los lleva a alinearse con los objetivos que proponemos, y a dedicar el esfuerzo necesario para alcanzarlos. No obstante, los resultados de los últimos 30-40 años de investigación sobre aprendizaje parece apuntar a que los mecanismos que orientan los procesos de aprendizaje de las personas matriculadas en nuestras asignaturas son demasiados complejos y son el resultado de la interacción de demasiadas variables como para poder ajustarse a modelos tan sencillos como los que sustentan las investigaciones de nivel 1. Por ello, la investigación que puede ser útil en estos momentos es aquella que se sitúa en el nivel 2, o mejor, en el nivel 3.

Como limitaciones de este trabajo, sólo hemos analizado las publicaciones del último año (2017) y de un subconjunto de los profesores universitarios españoles del área. Sería conveniente en una investigación futura, ampliar a las publicaciones de los últimos años (desde 2015) e incluir a una muestra más amplia de profesores (incorporando a los que pertenecen a la otra gran asociación científica del área, ADINGOR). Por otra parte, la identificación de artículos se ha realizado en base a las contestaciones de los autores potenciales. Este procedimiento puede haber dejado falsos negativos por no respuesta o despiste de los autores. En investigación futura se podría buscar las publicaciones de cada una de las personas adscritas a ADINGOR y ACEDEDOT en Web Of Science y Scopus y filtrar con los criterios de la Tabla 1. También se podría completar con una actualización de la revisión de Medina-López et al (Medina-López et al., 2011/d) restringiendo a los últimos años y autores españoles.

Entendemos que las reflexiones derivadas de los resultados no se pueden generalizarse al conjunto de investigación sobre aprendizaje realizado por profesores universitarios españoles sin realizar estudios similares en otras áreas de conocimiento.

\section{Referencias}

ALFALLA-LUQUE, R., GARRIDO-VEGA, P., DEL MAR GONZÁLEZ-ZAMORA, M., MEDINALÓPEZ, C., \& SACRISTÁN-DÍAZ, M. (2017). Un análisis sobre las causas de abandono de los trabajos fin de máster an analysis of the causes of master's thesis dropouts. Working Papers on Operations Management, 8, 32-49. doi:10.4995/wpom.v8i0.7114

ALHELY, B., CHÁVEZ, C., \& MIGUEL-DÁVILA, J.-Á. (2017). Experiencia docente de aplicación de kaizen en una empresa teaching experience of application of kaizen in a company. Working Papers on Operations Management, 8, 58-61. doi:10.4995/wpom.v8i0.7138

ÁLVAREZ-GIL, M. J., MONTES-SANCHO, M. J., \& TACHIZAWA, E. M. (2017). A first approximation to the spocs-fc in the context of the supply chain management. WPOM-Working Papers on Operations Management, 8(151-163). doi:10.4995/wpom.v8i0.7198

(cc) EY-NC-ND 2018, Universitat Politècnica de València

Congreso IN-RED (2018) 
ANDREU-ANDRÉS, M. A., MARÍN-GARCÍA, J. A., \& GARCÍA-CARBONELL, A. (2017). El barómetro incode como herramienta de autoevaluación de las competencias de innovación the barometer incode as a self-assessment tool of innovation competencies. REDU Revista de Educación Universitaria, 15(152), 275-294.

AVELLA CAMARERO, L. (2017). Estrechando los lazos universidad-empresas: Puesta en marcha del programa especialista en do y lean 6 sigma en respuesta a la demanda formativa de la industria. Working Papers on Operations Management, 8, 54-57. doi:10.4995/wpom.v8i0.7135

BIGGS, J., \& TANG, C. (2011 (1st edition 1999)). Teaching for quality learning at university. New York: McGraw-Hill Education, SRHE and Open University Press.

BLANCO, B., \& SANCHEZ-RUIZ, L. (2017). Fomentar el contacto con el mundo empresarial a través del trabajo fin de grado: Una experiencia aplicada a la temática de subcontratación encouraging contact with the business world through the final year project: An experience applied to the outsourcing field. Working Papers on Operations Management, 8, 62-66. doi:10.4995/wpom.v8i0.7159

BOYER, E. L. (1990). Scholarship reconsidered. Priorities of the professoriate. Princeton, NJ: Carnegie Foundation for the Advancement of Teaching.

BURKE-SMALLEY, L. A. (2014). Evidence-based management education. Journal of Management Education, 38(5), 764-767. doi:10.1177/1052562914529418

CANÓS-DARÓS, L., MORERA, C. P., SANTANDREU MASCARELL, C., PILAR, Y., \& VIDALCARRERAS, I. (2017). La evaluación de la enseñanza: Algunas reflexiones evaluation of teaching: Some reflections. Working Papers on Operations Management, 8, 67-71. doi:10.4995/wpom.v8i0.7163

CASCIO, W. F. (2007). Evidence-based management and the marketplace for ideas. Academy of management Journal, 50(5), 1009-1012. doi:10.5465/amj.2007.27151942

COFFEY, M., \& GIBBS, G. (2002). Measuring teachers' repertoire of teaching methods. Assessment and Evaluation in Higher Education, 27(4), 383-390. doi:10.1080/0260293022000001382

DE BURGOS JIMÉNEZ, J., FELIPE, J., GUERRERO, J., \& TARIFA FERNÁNDEZ, J. (2017). Creación de materiales didácticos para trabajos fin de estudios en gestión de la cadena de suministro creation of didactic materials for dissertation in supply chain management. Working Papers on Operations Management, 8, 19-26. doi:10.4995/wpom.v8i0.7107

DELBECQ, A. L. (2007). Scholarship and teaching that matters: Juxtaposing inner freedom with street-smart credibility. Journal of Management Education, 31, 376-391.

DÍAZ GARRIDO, E., MARTÍN-PEÑA, M. L., \& SÁNCHEZ-LÓPEZ, J. M. (2017). The impact of flipped classroom on the motivation and learning of students in operations management. WPOMWorking Papers on Operations Management, 8, 15-18. doi:10.4995/wpom.v8i0.7091

ENTWISTLE, N. (2000). Promoting deep learning through teaching and assessment. In L. Suskie (Ed.), Assessment to promote deep learning: Insight from aahe's 2000 and 1999 assessment conferences. (pp. 78). Washington: American Association for Higher Education

FERNÁNDEZ-ZAMORA, J. C., \& ARIAS-ARANDA, D. (2017). Implementation of a gamification platform in a master degree (master in economics). WPOM-Working Papers on Operations Management, 8, 181-190. doi:10.4995/wpom.v8i0.7431

FERNANDEZ MARCH, A. (2008). Training teachers in higher education. Teoria De La Educacion, 20, 275-312.

(c) 2018, Universitat Politècnica de València 
FERNÁNDEZ MARCH, A. (2010). La evaluación orientada al aprendizaje en un modelo de formación por competencias en la educación universitaria. Revista de Docencia Universitaria, 8(1), 11-34.

FOSSAS-OLALLA, M., MINGUELA-RATA, B., FERNÁNDEZ-MENÉNDEZ, J., \& GIMÉNEZFERNÁNDEZ, E. (2017). Experiencia de aprendizaje mediante el uso de una app en los estudios de grado learning experience using an app in bachelor degree. Working Papers on Operations Management, 8, 92-100. doi:10.4995/wpom.v8i0.7175

FRIESE, S. (2012). Qualitative data analysis with atlas.Ti. London: SAGE Publications Ltd.

GARCÍA-RAMOS, C., \& MARTÍNEZ-CAMPILLO, A. (2017). The service-learning methodology applied to operations management: From the operations plan to business start up. WPOM-Working Papers on Operations Management, 8(171-176). doi:10.4995/wpom.v8i0.7200

GIBBS, G., \& COFFEY, M. (2000). Training to teach in higher education: A research agenda. Teacher Development, 4(1), 31-44. doi:10.1080/13664530000200103

GUITART-TARRÉS, L., NÚÑEZ-CARBALLOSA, A., JARÍA CHACÓN, N., ACHCAOUCAOU, F., CRUZ-CÁZARES, C., MIRAVITLlES, P., \& HUERTAS GARCÍA, R. (2017). Playing in operations management. WPOM-Working Papers on Operations Management, 8, 81-85. doi:10.4995/wpom.v8i0.7171

LÓPEZ-SÁNCHEZ, J. I., FOSSAS-OLALLA, M., RODRÍGUEZ-DUARTE, A., \& SANDULLI, F. (2017). A new way to learn. The use of an app (blunder) to manage knowledge with higher education students. WPOM-Working Papers on Operations Management, 8(0), 86-91. doi:10.4995/wpom.v8i0.7174

MAQUEIRA MARÍN, J. M., MOYANO FUENTES, J., \& BRUQUE CÁMARA PEDRO NUÑEZCACHO UTRILLA, S. (2017). Diseño e implementación de una plataforma erp como entorno de aprendizaje en dirección de operaciones design and implementation of an erp platform as practice environment for learning in operations management. Working Papers on Operations Management, 8, 27-31. doi:10.4995/wpom.v8i0.7112

MARIMON, F., \& BERBEGAL-MIRABENT, J. (2017). Sinergias cruzadas entre el aprendizaje de contenidos propios de dirección de operaciones y el uso de herramientas de análisis genéricas cross learning synergies between operation management content and the use of generic analytic tools. Working Papers on Operations Management, 8, 13-14. doi:10.4995/wpom.v8i0.7015

MARIN-GARCIA, J. A. (2007). Taller sobre grounded theory y análisis cualitativo de datos con atlas.Ti

MARIN-GARCIA, J. A., MAHEUT, J., \& GARCIA-SABATER, J. J. (2017). Comparison of different ways of compute the grades of continuous assessment in the final grade. WPOM-Working Papers on Operations Management, 8(SpIss), in press.

MARTÍNEZ JURADO, P. J., \& MOYANO FUENTES, J. (2017). Aprendiendo a enseñar lean management mediante juegos: Revisión sistemática de la literatura learning to teach lean management through games: Systematic literature review. Working Papers on Operations Management, 8, 164170. doi:10.4995/wpom.v8i0.7199

MARTON, F., HOUNSELL, D., \& ENTWISTLE, N. (2005). The experience of learning. Implications for teaching and studying in higher education. 3rd (internet) edition. Edinburgh: University of Edinburgh, Centre for Teaching, Learning and Assessment.

MEDINA-LÓPEZ, C., ALFALLA-LUQUE, R., \& MARIN-GARCIA, J. A. (2011). Research in operations management teaching: Trends and challenges. Intangible Capital, 7(2), 507-548.

(cc) EY-NC-ND 2018, Universitat Politècnica de València

Congreso IN-RED (2018) 
MORRELL, K., \& LEARMONTH, M. (2015). Against evidence-based management, for management learning. Academy of Management Learning \& Education, 14(4), 520-533. doi:10.5465/amle.2014.0346

OLTRA MESTRE, M. J., GARCIA PALAO, C., \& FLOR, M. (2017). Análisis de procesos del trabajo en equipo como recurso docente en los cursos de dirección de operaciones teamwork process analysis as a teaching resource in operations management courses. Working Papers on Operations Management, 8, 76-80. doi:10.4995/wpom.v8i0.7170

PARICIO ROYO, J. (2013). ¿repensar la formación del profesorado?¿porqué habríamos de hacer tal cosa? REDU: Revista de Docencia Universitaria, 11(3).

PARICIO ROYO, J. (2017). Los focos de la investigación en educación superior, Valencia (Spain).

PARICIO ROYO, J., \& ALLUEVA PINILLA, A. I. (2011). Prácticas y modelos innovadores para la mejora y calidad de la docencia: Prensas Universitarias de Zaragoza.

PEARCE, J. L. (2007). We are who we teach - how teaching experienced managers fractures our scholarship. Journal of Management Inquiry, 16(2), 104-110. doi:10.1177/1056492607302412

RAMÍREZ, F. J., \& GARCÍA-VILLAVERDE, P. M. (2017). Ddi tool: Un juego serio para el desarrollo de las competencias de los estudiantes de grado y postgrado en el entorno de la dirección de operaciones ddi tool: A serious game for the development of competences of graduate and postgraduate students in the operations management environment. Working Papers on Operations Management, 8, 50-53. doi:10.4995/wpom.v8i0.7127

RAMSDEN, P. (1992). Learning to teach in higher education: Routledge.

ROSILLO, R., ÁNGEL, J., DÁVILA, M., ROBLES, C. F., \& REDONDO, M. M. (2017). Sistema de innovación docente aplicado en asignaturas de gestión de empresas teaching innovation system applied in business management subjects. Working Papers on Operations Management, 8, 143-150. doi:10.4995/wpom.v8i0.7195

SANCHEZ-RUIZ, L., BLANCO, B., \& MARÍN-GARCÍA, J. A. (2017). Protocolo: Validación del barómetro incode para medir la competencia de innovación con la teoría de medición de rasch protocol: Validation of the incode barometer to measure the innovation competence through the rasch measurement theory. Working Papers on Operations Management, 8, 120-124. doi:10.4995/wpom.v8i0.7190

VIDAL-CARRERAS, P. I., GARCIA-SABATER, J. J., \& CANOS-DAROS, L. (2017). Una propuesta para la disciplina de dirección de operaciones en el grado en ingeniería electrónica industrial y automática a proposal for a course of operations management in the degree in industrial electronic and automatic engineering. Working Papers on Operations Management, 8, 177-180. doi:10.4995/wpom.v8i0.7205

VIDAL, J. V., MARTINEZ, P. C., \& ALFARO, J. A. (2017). Experiences of teaching innovation for the consolidation of a r\&d\&i culture. WPOM-Working Papers on Operations Management, 8, 125139. doi:10.4995/wpom.v8i0.7191 